\title{
Adaptive Smoothed Particle Hydrodynamics for Study of Friction of Silica at Micronscale
}

\author{
Le Van Sang ${ }^{1)^{*}}$, Akihiko Yano ${ }^{2)}$, Ai Osaka ${ }^{2)+}$, Natsuko Sugimura ${ }^{1,4)}$ and Hitoshi Washizu ${ }^{1,3)^{* *}}$ \\ ${ }^{1)}$ Graduate School of Simulation Studies, University of Hyogo, 7-1-28 Minatojima Minamimachi, Chuo-ku, Kobe, Hyogo 650-0047, Japan \\ ${ }^{2)}$ Mitsubishi Heavy Industries, Ltd., 2-1-1 Arai-cho, Shinhama, Takasago, Hyogo 676-8686, Japan \\ ${ }^{3)}$ Elements Strategy Initiative for Catalysts and Batteries (ESICB), Kyoto University, 1-30 Goryo-Ohara, Nishikyo-ku, Kyoto 615-8245, Japan \\ ${ }^{4)}$ Faculty of Engineering, Tokyo City University, 1-28-1 Tamazutsumi, Setagaya, Tokyo 158-8557, Japan \\ 'Present address: The Graduate Institute of Scientific and Industrial Research, Osaka University, 8-1 Mihogaoka, Ibaraki, Osaka 567-0047, Japan
}

\author{
*Corresponding author: Le Van Sang (levansang82@gmail.com)
}

${ }^{* *}$ Corresponding author: Hitoshi Washizu (h@washizu.org)

Manuscript received 28 April 2020; accepted 29 July 2020; published 31 August 2020

\begin{abstract}
The paper focuses on examining agreement of the adaptive smoothed particle hydrodynamics (ASPH) in the investigation of the sliding friction of silica at micronscale throughout observation of several friction characteristics. It is found that the ASPH approach well presents the friction of micronscale silica due to agreement of the friction coefficient and the applied load-friction coefficient relationship between the present results and the previously experimental reports. The shape of the particle modeled in the ASPH almost does not effect on the detected results for the hard system due to the very slight variation of the particles during the sliding. However, the variation of the particles can explain for the discrepancy between the stick time and the slip time and the unsharp change between the stick state and the slip one. The study is also extended for the contacts of the two sinusoidal rough surfaces and finds that the friction coefficient is almost independent of the wavelength while it linearly increases with the amplitude.
\end{abstract}

\section{Keywords}

sliding friction, silica contact, micronscale, stick-slip time, ASPH method, particle discretization

\section{Introduction}

The smoothed particle hydrodynamics (SPH) approach or the ASPH approach has been widely used to investigate the micronscale system because it provides the discretization of the continuous system. The main difference between the two approaches is that the particles interact with each other throughout the isotropic kernel in the SPH approach and with the anisotropic kernel in the ASPH approach. Therefore, in order to investigate the influences of the particle shape on the properties of the micronscale materials, one should carry out the ASPH calculations for those. The ASPH method also showed the better time evolution of the density profiles [1] and the high strain hydrodynamic problems [2] than did the SPH method. Many studies reported the effects of the particle shape on the different properties of materials in the sizes of from micro to macroscale. Smeets et al. established the contact force interactions between the polyhedral particles in the discrete element method (DEM) and simulated the particle flow to compare the packing factors of the different shapes [3]. The shape of the third body was found to strongly result in the sliding friction of the contact [4]. The particles in these studies were considered to freely separate to each other instead of the grid of the particles that has been widely used to discretize the solid system in the methods as the SPH, the ASPH and the FEM (finite element mothod). Other research groups have modeled the atoms as the spherical particles or the ellipsoidal particles by developing the interaction potentials $[5,6]$. The anisotropic forces can well describe the interaction between the coarsegrained particles that are lumped from a materials region of comprising several different elements. In this case, the coarsegrained particle can show the different interaction strengths in the different directions due to its heterogeneous situation. Therefore, these coarse-grained systems can be suitable to be modeled by the ASPH approach instead of the SPH one.

This study investigates the dry sliding friction of the micronscale $\alpha-\mathrm{SiO}_{2}$ oxide by employing with the discretization and the ASPH simulations. The friction, adhesion and wear properties of $\mathrm{SiO}_{2}$ oxide should be extensively investigated because of its common applications in the multilayer semiconductor devices and its dominant presence in the rocks whose unstable movement is related to the earthquake 
phenomenon. The different contributions to the friction of $\mathrm{SiO}_{2}$ oxide have been reported as the thermal and quantum effects on the Casimir frictional drag force [7]. There were also the discussions of the friction of quartz related to the earthquake phenomenon $[8,9]$. This study focuses on detecting the sliding friction of the micronscale silica with the smooth contact and the contacts of the two sinusoidal rough surfaces. Several shapes of the silica particles modeled throughout the anisotropy of the kernel in the ASPH approach are considered and discussed.

\section{Method and simulation}

In the ASPH method, the time evolutions of the density $(\rho)$, the velocity $(\vec{v})$ and the internal energy $(u)$ of the particle are described as

$$
\begin{aligned}
& \frac{d \rho_{i}}{d t}=\sum_{j=1}^{N} m_{j}\left(\vec{v}_{j}-\vec{v}_{i}\right) \vec{\nabla}_{i} W\left(\vec{\eta}_{i}\right), \\
& \frac{d v_{i}^{\alpha}}{d t}=\sum_{j=1}^{N} m_{j}\left(\frac{\sigma_{i}^{\alpha \beta}}{\rho_{i}^{2}}+\frac{\sigma_{j}^{\alpha \beta}}{\rho_{j}^{2}}+\Pi_{i j}\right) \nabla_{i}^{\beta} W\left(\vec{\eta}_{i}\right), \\
& \frac{d u_{i}}{d t}=\frac{1}{2} \sum_{j=1}^{N} m_{j}\left(\frac{\sigma_{i}^{\alpha \beta}}{\rho_{i}^{2}}+\frac{\sigma_{j}^{\alpha \beta}}{\rho_{j}^{2}}+\Pi_{i j}\right)\left(v_{j}^{\alpha}-v_{i}^{\alpha}\right) \nabla_{i}^{\beta} W\left(\vec{\eta}_{i}\right),
\end{aligned}
$$

where $\alpha, \beta \equiv x, y, z ; \vec{\eta}_{i}=G_{i} \vec{r}_{i j}$ with $\vec{r}_{i j}=\vec{r}_{i}-\vec{r}_{j}$ and $G_{i}$ is the second order tensor; the Gauss kernel $W$ is as that in Ref. 1 ; the stress tensor $\sigma$ and the artificial viscosity function $\Pi$ are as those in Ref. 10 . The time evolution of the $G$ tensor is described by $d G / d t=I^{G} G-G \sigma^{G}$, where the second order tensors $\sigma^{G}$ and $I^{G}$ are as those in Ref. 1 . In the primary frame of the kernel, the $G$ tensor is described as

$$
G^{(k)}=\left(\begin{array}{ccc}
G_{11}^{(k)} & 0 & 0 \\
0 & G_{22}^{(k)} & 0 \\
0 & 0 & G_{33}^{(k)}
\end{array}\right)
$$

In general, $G_{11}^{(k)} \neq G_{22}^{(k)} \neq G_{33}^{(k)}$ in the ASPH method. The full rotational transformation matrices $T_{\vec{r}}(\phi, \Psi, \theta)$ (as that in Ref. 1) are used to transform the tensor of the kernel primary frame $\left(G^{(k)}\right)$ to that of the real frame $\left(G^{(r)}\right)$ as

$$
G^{(r)}=T_{\vec{r}}^{T}(\phi, \psi, \theta) G^{(k)} T_{\vec{r}}(\phi, \psi, \theta),
$$

where $T_{\vec{r}}^{T}$ is the transposed matrix of the $T_{\vec{r}}$ matrix. The size and the anisotropy of the particle are modeled by $G_{11}^{(k)}, G_{22}^{(k)}$, $G_{33}^{(k)}$; and its orientation is modeled by $\phi, \Psi$ and $\theta$. Note that, we describe the briefed presentations of the calculations of the above quantities in this study. One can also see the detailed representation of $\mathrm{W}\left(\vec{\eta}_{i}\right), \Pi_{i j}, \sigma_{i}^{\alpha \beta}, \sigma^{G}, \Gamma^{G}$ and $T_{\vec{r}}$ in the Appendix.

The spring force presenting the interaction between the interfacial particles, one of the lowest layer of the slider and the other of the highest layer of the substrate, is described as

$$
\vec{F}_{i n t, i j}=\left\{\begin{array}{c}
-K_{\alpha}\left(r_{i j}-r_{c u t}\right) \frac{\vec{r}_{i j}}{r_{i j}}, 0<r_{i j} \leq r_{c u t} \\
0, \quad r_{i j}>r_{c u t}
\end{array}\right.
$$

where $K_{\alpha}$ is the spring constant. For the micronscale or macroscale contacts, the spring force has been found to well present the interaction between the interfacial coarse-grained particles such as the particles of solid rocks, iron, alumina and hematite in the previous studies of the sliding friction [10-13]. In order to apply the Prandtl-Tomlinson model, the particles of the slider are acted by other spring force as

$$
\vec{F}_{P T, i}=K\left(\vec{r}_{0, i}+\vec{V}_{s} t-\vec{r}_{i}\right)
$$

where $K, t, \vec{r}_{0}$ and $\vec{V}_{s}\left(\vec{V}_{s}=\left(V_{s}, 0,0\right)\right)$ are the spring constant, the sliding time, the initial position vector and the sliding velocity of the slider along the $x$-direction, respectively. The slider is pushed as one unit with the $V_{s}$ velocity at the start of the sliding after which only the particles of the highest layer are held at this velocity during the sliding. The friction coefficient $(\mathrm{CoF}) \mu_{\text {cof }}$ is defined as

$$
\mu_{c o f}=\frac{\sum_{i=1}^{N_{f}}\left(F_{P T, i}^{x}+F_{i n t, i j}^{x}\right)}{\sum_{i=1}^{N_{f}}\left(F_{P T, i}^{z}+F_{i n t, i j}^{z}\right)}
$$

where $N_{f}$ is the number of the particles (the friction particles) of the contact layer of the slider. The numerator and the denominator of Eq. (8) are defined as the friction force and the normal force, respectively. The dissipation force acting on each particle of the systems to compensate for the energy dissipation caused by the friction is described as

$$
\vec{F}_{d i s, i}=-m_{i} \gamma_{d i s}\left(\vec{v}_{i}-\vec{V}_{i n}\right),
$$

where $\gamma_{\text {dis }}$ is the dissipation parameter of the model and $\vec{V}_{\text {in }}$ is the initial velocity of the particles. Note that the particle is added the accelerations (Eq. (2)) by the forces in Eqs. (6), (7) and (9).

The discretization of the atomistic $\alpha-\mathrm{SiO}_{2}$ system is done by lumping the volume of the atomistic system into a particle (Fig. 1a). A discretized particle system is obtained by periodically spreading out an origination particle along the three dimensions. The simulated particle system including the 9,000 particle slider with the sizes of $33.7306 \times 21.3839 \times 6.6356$ $\mu^{3}$ and the 40,000 particle substrate with the sizes of $79.1151 \times$ $36.1315 \times 6.6356 \mu \mathrm{m}^{3}$ is described in Fig. 1b. Figure 2 describes two of the simulated systems with the contacts of the sinusoidal rough surfaces. The initial distance between the slider and the substrate is set equal to $\left|\vec{t}_{c}\right|$. The lowest particle layer of the substrate is fixed during the simulations. The simulation parameters, some of which are seen in the Appendix, are set as
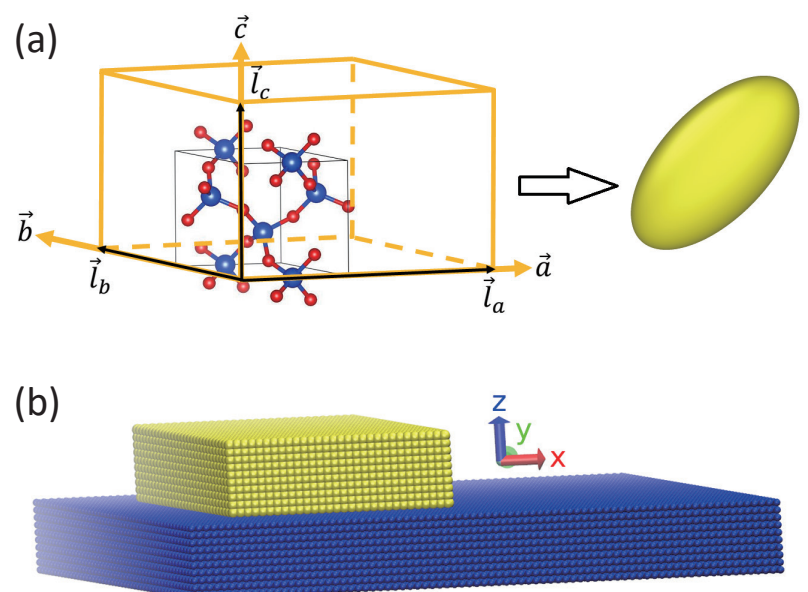

Fig. 1 The process of lumping of the volume of the atomistic $\alpha-\mathrm{SiO}_{2}$ yielded by the three vectors $\vec{l}_{a}, \vec{l}_{b}$ and $\vec{l}_{c}$ into the particle, where $\left(\vec{l}_{a}, \vec{l}_{c}\right)=\left(\vec{l}_{b}, \vec{l}_{c}\right)=90^{\circ},\left(\vec{l}_{a}, \vec{l}_{b}\right)=120^{\circ},\left|\vec{l}_{a}\right|=$ $\left|\vec{l}_{b}\right|=\left|\vec{l}_{c}\right|=0.7374 \mu \mathrm{m}$ and the mass of the particle of $m$ $=1.061 \times 10^{-15} \mathrm{~kg}(\mathrm{a})$; and the simulated particle system with the smooth contact including the 9,000-particle slider (yellow color) and the 40,000-particle substrate (blue color) (b). 
(a)

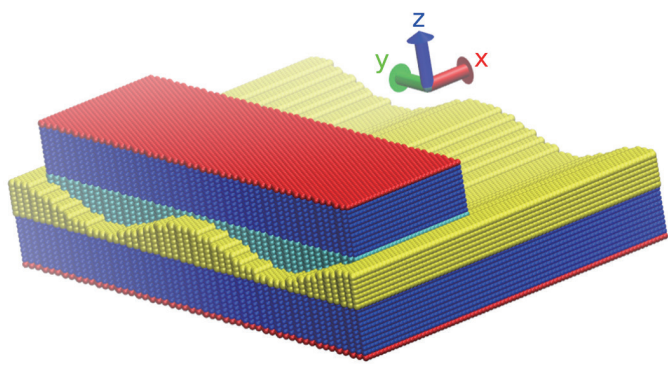

(b)

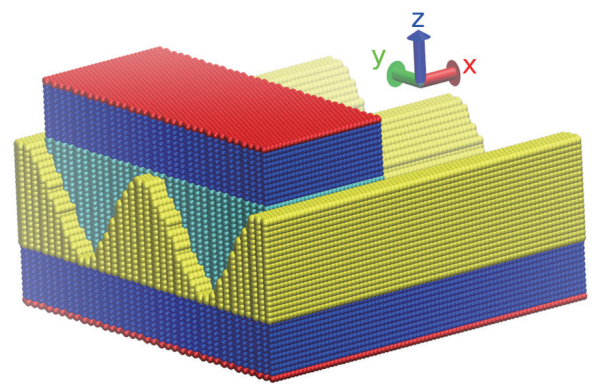

Fig. 2 Two of the simulated particle systems with the contacts of the two sinusoidal rough surfaces, $z=N_{a m}\left|\vec{l}_{c}\right| \sin$ $\left(\frac{2 \pi y}{N_{w l} \mid \vec{l}_{c} l}\right)$, with the particle number of the slider, the particle number of the substrate, $N_{a m}$ and $N_{w l}$ corresponding to 145440,50040, 3 and 60 (a) and 146400, 48600, 9 and 40 (b). The particles are colored by the following reasons: the clear view for the slider (the higher three colors) or the substrate (the lower three colors), the parts of the sinusoidal roughness (yellow and cyan colors), the lower red colored part is fixed during the simulation and the higher red colored part is directly acted by the externally applied load.

follows: $V_{s}=50 \mathrm{~m} / \mathrm{s}, d t=100$ ps (the simulation time step), $\rho=$ $2648 \mathrm{~kg} / \mathrm{m}^{3}, \mu=46.91 \mathrm{GPa}$ (the shear modulus) [14], $\alpha_{W}=0.1, h_{c r}$ $=1.4, \alpha_{\Pi}=2, \beta_{\Pi}=5, \gamma_{\text {dis }}=10^{9} 1 / \mathrm{s}, r_{\text {cut }}=\sqrt{2}\left|\vec{l}_{a}\right|, K=0.104 \mathrm{~N} / \mathrm{m} \mathrm{[15],}$ and $K_{x}=K_{y}=K_{z}=0.2 \mathrm{~K}[10]$. The ASPH simulation program is modified from the FDPS open source, which was developed by the Particle Simulator Research Team (AICS, RIKEN, JAPAN) [16]

\section{Results and discussion}

Figure 3 describes the variations of the friction force and the friction coefficient in the sliding distance at the applied load of $10 \mu \mathrm{N}$ for the two initial shapes of the particles. These variations are clear independent of the particle shape. Therefore, spreading out the interaction region of the kernel or making the particle size larger in the normal direction does not result in the friction characteristics. This is due to the hardness of the contact surfaces and the slight influence of the inner particles on the friction. The contact surfaces (or the slider and the substrate) are hardly held throughout the dissipation parameter. The inner particles very slight result in the friction due to the contact layers of the $0.7374 \mu \mathrm{m}$ thickness. This is agreeable with the widely known findings that only several atomic layers of the contact surfaces result in the friction. The stick-slip friction regularly occurs during the sliding with the longer stick time than the slip time, seen clearly in the inserted figure in Fig. 3a. The ratio between these two times is equal to 2.0 in this study. The different ratios of these two times have been
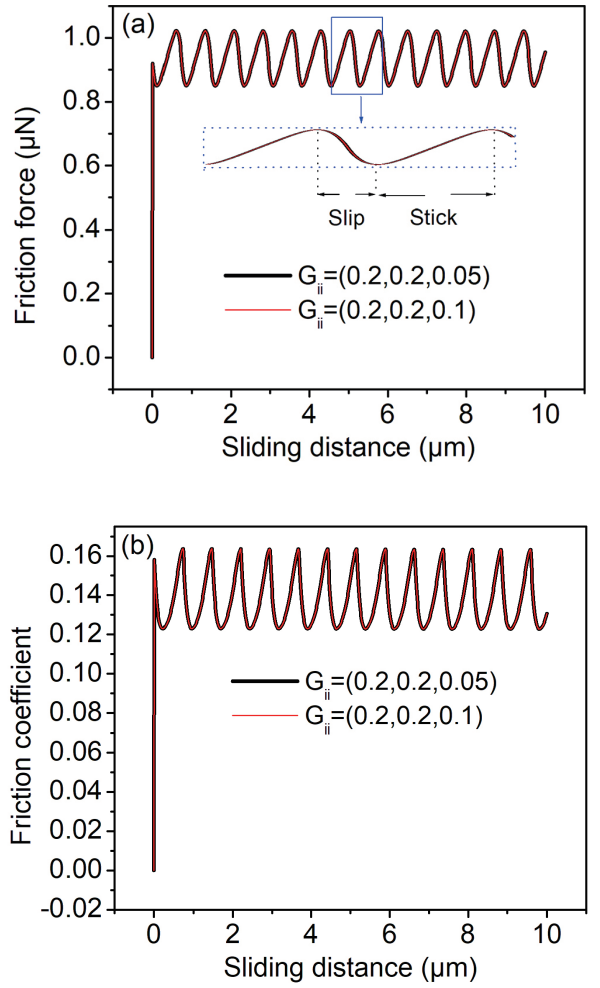

Fig. 3 The variations of the friction force (a) and the friction coefficient (b) in the sliding distance for the two initial shapes of the particle: $\left(G_{11}^{(k)}, G_{12}^{(k)}, G_{13}^{(k)}\right)(1 / \mu \mathrm{m})$ given in the figure and $(\phi, \Psi, \theta)=(0,0,0)$.

experimentally reported for the stick-slip friction of $\mathrm{CrVN}$ at the two temperatures as 9.0 at $25^{\circ} \mathrm{C}$ and 2.3 at $500^{\circ} \mathrm{C}$ [17]. The back and forth jumps of the tip between the two atomic positions before finally settling at the new position were found to be the possible mechanisms causing the longer slip duration [18]. This study indicates that one can observe the longer stick time than the slip time throughout the the anisotropy of the particle shape modeled by the kernel in the ASPH method. This anisotropy can be also figured out as the jumps of the tip due to the variation of the interaction. This situation has not been observed in the previous studies for the sliding friction of various materials by using the SPH method [10-13]. Therefore, the anisotropy of the particle shape can be the main reason causing this result. Due to the interaction between the ellipsoidal particles, the exchange between the stick and the slip does not also sharply occur like that commonly found for the stick-slip friction of the point interaction systems. This study does not find any dependence of the above ratio on the particle shape. The CoF oscillates from 0.1228 to 0.1634 in the steady interval, with the average value of 0.1376 (Fig. 3b). This result is comparable with the CoF of $\mathrm{SiO}_{2}$ contacts reported in the previously experimental studies. The static and the dynamic CoFs of the clear quartz, the milky quartz and the rose quartz were found to be 0.11 and $0.10,0.14$ and 0.14 , and 0.13 and 0.11 under the over-dried condition; or 0.11 and $0.10,0.16$ and 0.16 , and 0.13 and 0.11 under the overdried/air-equilibrated condition, respectively [19]. Additionally, the $\mathrm{SiO}_{2}$ tip/the $\mathrm{SiO}_{2}$ flat contact showed the static $\mathrm{CoF}$ of 0.20 or 0.15 at the different experimental pressures [20].

As seen in Fig. 4, the average CoF linearly decreases from 0.1379 to 0.1341 in the applied load range of $5-80 \mu \mathrm{N}$. Due to 


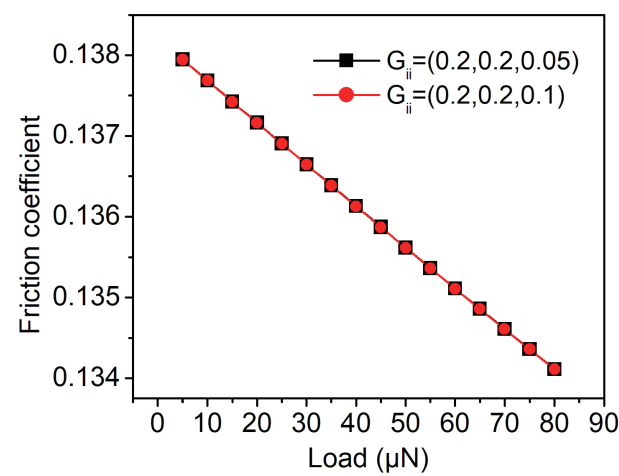

Fig. 4 The variations of the friction coefficient in the applied load for the two initial shapes of the particle: $\left(G_{11}^{(k)}, G_{12}^{(k)}\right.$,

$\left.G_{13}^{(k)}\right)(1 / \mu \mathrm{m})$ given in the figure and $(\phi, \Psi, \theta)=(0,0,0)$.

the very slight reduction of the $\mathrm{CoF}$, it can be considered to be stably maintained in this applied load range. This is due to the very slight deformation of the silica contact surfaces at the low applied load. Similarly, Kumar et al. experimentally reported that the dynamic CoF of the fused silica sample derived by using the $20-\mu \mathrm{m}$ conical indenter is constant at around 0.14 in the applied load range of $0-2000 \mu \mathrm{N}$ [21]. It is worth noting that the applied load-CoF relationship strongly depends on the indenter size [21]. The size of the slider in this study is close to $20 \mu \mathrm{m}$ in the $x$ - or $y$-direction; therefore, the present result is agreeable with the experimental observation [21]. The experimentally dynamic $\mathrm{CoF}$ of the $\mathrm{SiO}_{2} / \mathrm{SiO}_{2}$ millimeter scale smooth contact showed the reduction in the ultrahigh vacuum pressure range of $10^{-2}-10^{-7} \mathrm{~Pa}$ and the increase in the range of $<$ $10^{-7} \mathrm{~Pa}$; however, it oscillates from 0.15 to 0.22 in both the cases [22]. As a summary, the applied load-CoF relationship shows the similar value or the similar change for the $\mathrm{SiO}_{2}$ contacts of the different sizes as micronmeter (the present results), millimeter [20] and centimeter [19, 22].

Figure $5 \mathrm{a}$ describes the variation of the $\mathrm{CoF}$ in the sliding distance at the applied load of $10 \mu \mathrm{N}$ for the six orientations of the particles. The orientation of the particle almost does not result in the sliding distance dependence of the CoF due to the similarity of the curves. Therefore, both the size and orientation of the kernel (or particle) almost do not result in the characteristics of the sliding friction of the stable system. This situation is due to the slight change of the kernel (Fig. 5b) in the stable system leading to the fact that the interaction between the particles slightly varies during the sliding. The ASPH method has produced the good results of the highly confused systems [2]; therefore, it can be appropriate to use this method for the tribological studies of the confused systems including the collision of the rough contacts or the asperities. It is also worth recalling that the ASPH method still well presents the sliding friction of the steady system, as seen in this study. Additionally, Framework for Developing Particle Simulator (FDPS) [16] is suitable for massive parallel calculation; therefore, this method can be used to simulate the realistic friction phenomena with the realistic surface roughness. Although the variation of the kernel does not result in the CoF, it can explain for the discrepancy between the stick time and the slip time and the unsharp change between the stick and the slip.

Figure 6 describes the variation of the $\mathrm{CoF}$ in the sliding distance at the applied load of $10 \mu \mathrm{N}$ and the wave amplitude for the contacts of the sinusoidal rough surfaces. As seen in
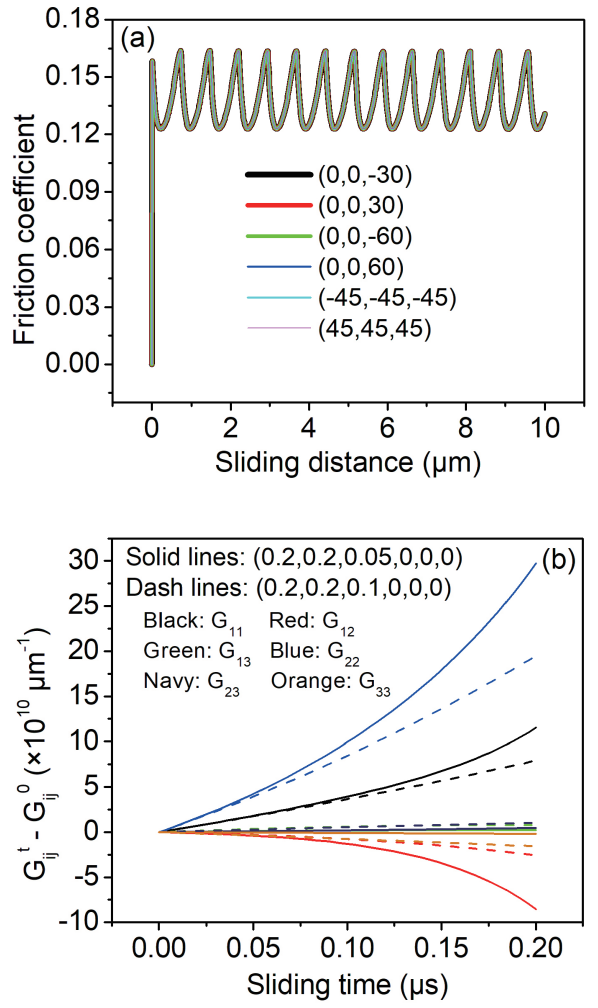

Fig. 5 The variation of the friction coefficient in the sliding distance with the initial behaviors $\left(G_{11}^{(k)}, G_{12}^{(k)}, G_{13}^{(k)}\right)=$ $(0.2,0.2,0.05)(1 / \mu \mathrm{m})$ and $(\phi, \Psi, \theta)$ (degree) given in the figure (a) and the time evolution of the $G$ tensor for the two initial behaviors with $\left(G_{11}^{(k)}, G_{12}^{(k)}, G_{13}^{(k)}, \phi, \Psi, \theta\right)(1 / \mu \mathrm{m}$, degree) given in the figure (b).
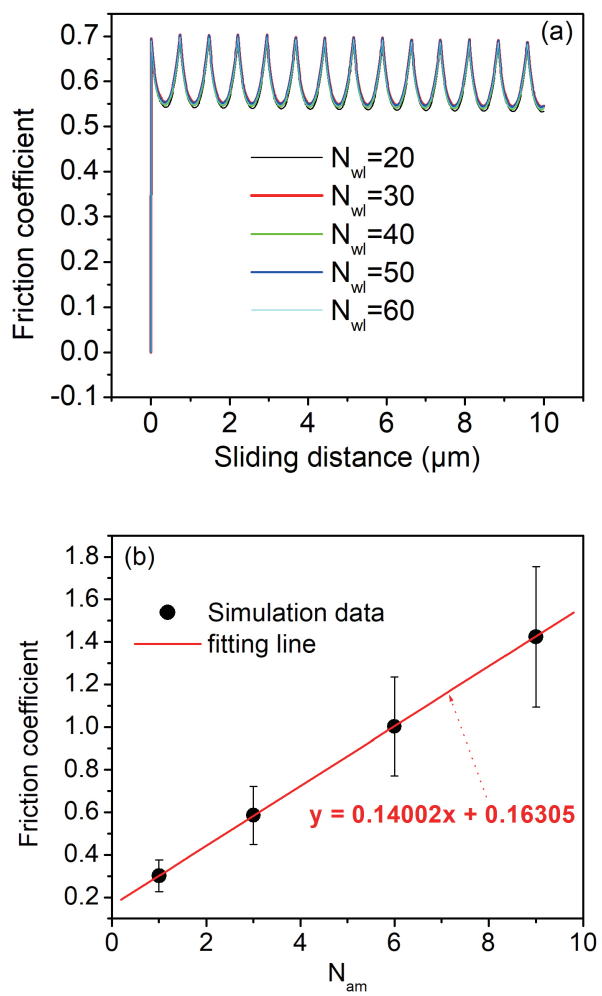

Fig. 6 The variations of the friction coefficient on the wavelength with $N_{a m}=3$ (a) and the amplitude with $N_{w l}=40$ (b). 
Fig. 6a, at the given amplitude the CoF very slightly depends on the wavelength, having the maximum value of 0.689 and the minimum value of 0.541 for all cases. This result can be comparable with the $\mathrm{CoF}$ of the quartz gouge. The steady state CoF of the quartz gouge has been experimentally found to be varying from 0.66 to 0.69 [23] or the order of 0.6 [24]. The CoF showed the increase with the percent of the quartz in the montmorillonite-quartz simulated gouge and reached the stable value of around 0.5 as the quartz occupied from 85 to $100 \%$ [25]. With $N_{a m}=3$, the contact between the surfaces is not more deeply; therefore, the contact in this study can be almost similarly to that of the quartz gouge systems in the experimental studies. This leads to the similarity of the CoF. In contrast to its dependence in the wavelength, the CoF shows the linear increase with the amplitude at the given wavelength (Fig. 6b). By extrapolating from the equation presenting the fitting line of the simulation data (Fig. $6 \mathrm{~b}$ ), the estimated $\mathrm{CoF}$ is equal to 0.163 as the amplitude of the sinusoidal roughness approaches zero. This result is consistent with the friction observations of the smooth contact discussed the above in which the averaged $\mathrm{CoF}$ is equal to 0.1376 . As is well known, the friction coefficient shows the higher value with the larger roughness of the contact surfaces [26]. Therefore, these observations can be explained by the rough situation of the contact surfaces. In the case of the fixed amplitude, the roughness shows the slight variation with the increase in the wavelength. In the other case, the roughness significantly increases with the increase in the amplitude due to the depth of the contact part and many rough particle layers occurring inside the contact. Additionally, the strong increase of the $\mathrm{CoF}$ with the amplitude is also because the fact that the slider sinks more deeply into the substrate. This is similar to the more depth of the intender in the substrate, which has been widely known to result in the increase of the CoF.

\section{Conclusions}

In summary, this study shows that the ASPH method can well present the sliding friction of micronscale $\mathrm{SiO}_{2}$ throughout the observations and the comparisons of the present results with the previous reports. The shape of the particles very slightly varies during the sliding for the hard contact surfaces leading the fact that the friction properties almost do not depend on it. However, the variations of the particle shape can explain for the discrepancy between the stick time and the slip time and the unsharp change between the stick and the slip. For the contacts of the two sinusoidal rough surfaces, the CoF does not depend on the wavelength while it linearly increases with the amplitude due to the roughness formed inside the contact and the more deep sink of the slider on the substrate.

\section{Acknowledgements}

This work was supported by JSPS KAKENHI Grant Numbers JP18K18813, JP19H05718 and JP20K04245.

\section{Appendix}

The Gauss kernel - The Gauss kernel and its derivate are defined as

$$
W\left(\vec{\eta}_{i}\right)=\frac{\alpha_{W}^{3 / 4} / G_{i} /}{\pi \Gamma(3 / 4)} \exp \left(-\alpha_{W} \dot{\eta}_{i}^{4}\right)
$$

$$
\vec{\nabla} W\left(\vec{\eta}_{i}\right)=-4 \alpha_{W} W\left(\vec{\eta}_{i}\right) \vec{\eta}_{i}^{2} G_{i} \vec{\eta}_{i}
$$

In all the calculations, $W\left(\vec{\eta}_{i}\right)$ and $\vec{\nabla} W\left(\vec{\eta}_{i}\right)$ are replaced by $W_{i j}=0.5$ $\left[W\left(\vec{\eta}_{i}\right)+W\left(\vec{\eta}_{j}\right)\right]$ and $\vec{\nabla} W_{i j}=0.5\left[\vec{\nabla} W\left(\vec{\eta}_{i}\right)+\vec{V} W\left(\vec{\eta}_{j}\right)\right]$, respectively. The artificial viscosity function - This is defined as

$$
\Pi_{i}= \begin{cases}\rho_{i}^{-1}\left(-\alpha_{\Pi} c_{i} \mu_{i}+\beta_{\Pi} \mu_{i}^{2}\right), & \vec{v}_{i j} \cdot \vec{r}_{i j}<0 \\ 0, & \vec{v}_{i j} \cdot \vec{r}_{i j} \geq 0\end{cases}
$$

where $\alpha_{\Pi}$ and $\beta_{\Pi}$ are the parameters; $c_{i}=\sqrt{h_{c r} P_{i} / \rho_{i}}$ is the speed of sound of the particle with the pressure of the particle $p_{i}=\left(h_{c r}-1\right) \rho_{i} u_{i}$ in which $h_{c r}$ is the parameter; $\mu_{i}=\vec{v}_{i j} \cdot \vec{\eta}_{i} /\left(\vec{\eta}_{i} \cdot \vec{\eta}_{i}+\xi^{2}\right)$ in which $\xi$ is the very small parameter for avoiding numerical error. In all the calculations, $c_{i}$ and $\Pi_{i}$ are replaced by $c_{i j}=0.5\left(c_{i}\right.$ $\left.+c_{j}\right)$ and $\Pi_{i j}=0.5\left(\Pi_{i}+\Pi_{j}\right)$, respectively.

The stress tensor - This is defined as

$$
\sigma_{i}^{\alpha \beta}=-p_{i} \delta^{\sigma \beta}+S_{i}^{\alpha \beta}
$$

where $\delta^{\sigma \beta}$ is Kronecker symbol and $S_{i}^{\alpha \beta}$ is the deviatoric stress that is calculated from the following equation

$$
\frac{d S_{i}^{\alpha \beta}}{d t}=2 \mu\left(\dot{\varepsilon}_{i}^{\alpha \beta}-\frac{1}{3} \delta^{\sigma \beta} \dot{\varepsilon}_{i}^{\gamma \gamma}\right)+S_{i}^{\alpha \gamma} R_{i}^{\beta \gamma}+S_{i}^{\gamma \beta} R_{i}^{\alpha \gamma}
$$

in which $\mu, \dot{\varepsilon}_{i}^{\alpha \beta}$ and $R_{i}^{\alpha \beta}$ are the shear modulus of materials, the tensor of the rate of deformations and the tensor of stress rotation, respectively, which are defined as

$$
\begin{aligned}
& \dot{\varepsilon}_{i}^{\alpha \beta}=\frac{1}{2} \sum_{j=1}^{N} \frac{m_{j}}{\rho_{j}}\left[\left(v_{j}^{\alpha}-v_{i}^{\alpha}\right) \nabla_{i}^{\beta} W\left(\vec{\eta}_{i}\right)+\left(v_{j}^{\beta}-v_{i}^{\beta}\right) \nabla_{i}^{\alpha} W\left(\vec{\eta}_{i}\right)\right], \\
& R_{i}^{\alpha \beta}=\frac{1}{2} \sum_{j=1}^{N} \frac{m_{j}}{\rho_{j}}\left[\left(v_{j}^{\alpha}-v_{i}^{\alpha}\right) \nabla_{i}^{\beta} W\left(\vec{\eta}_{i}\right)-\left(v_{j}^{\beta}-v_{i}^{\beta}\right) \nabla_{i}^{\alpha} W\left(\vec{\eta}_{i}\right)\right] .
\end{aligned}
$$

The second order tensors and the full rotational transformation matrices - Those are defined as

$$
\begin{aligned}
\sigma^{G} & =\left(\begin{array}{lll}
\sigma_{11}^{G} & \sigma_{12}^{G} & \sigma_{13}^{G} \\
\sigma_{21}^{G} & \sigma_{22}^{G} & \sigma_{23}^{G} \\
\sigma_{31}^{G} & \sigma_{32}^{G} & \sigma_{33}^{G}
\end{array}\right)=\left(\begin{array}{lll}
\partial v_{x} / \partial x & \partial v_{x} / \partial y & \partial v_{x} / \partial z \\
\partial v_{y} / \partial x & \partial v_{y} / \partial y & \partial v_{y} / \partial z \\
\partial v_{z} / \partial x & \partial v_{z} / \partial y & \partial v_{z} / \partial z
\end{array}\right), \\
\Gamma_{G} & =\left(\begin{array}{lll}
\Gamma_{11}^{G} & \Gamma_{12}^{G} & \Gamma_{13}^{G} \\
\Gamma_{21}^{G} & \Gamma_{22}^{G} & \Gamma_{23}^{G} \\
\Gamma_{31}^{G} & \Gamma_{32}^{G} & \Gamma_{33}^{G}
\end{array}\right)=\left(\begin{array}{ccc}
0 & \Gamma_{12}^{G} & -\Gamma_{31}^{G} \\
-\Gamma_{12}^{G} & 0 & \Gamma_{23}^{G} \\
\Gamma_{31}^{G} & -\Gamma_{23}^{G} & 0
\end{array}\right),
\end{aligned}
$$

where

$$
\frac{\partial v_{i}^{\alpha}}{\partial r_{i}^{\beta}}=\sum_{j=1}^{N} \frac{m_{j}}{\rho_{j}}\left(v_{j}^{\alpha}-v_{i}^{\alpha}\right) \nabla_{i}^{\beta} W\left(\vec{\eta}_{i}\right)
$$

$$
\Gamma_{12}^{G}=\frac{\gamma_{c} \gamma_{d}-\gamma_{b} \gamma_{e}}{\gamma_{a} \gamma_{c}-\gamma_{b}^{2}},
$$

$$
\Gamma_{31}^{G}=\frac{\gamma_{b} \gamma_{d}-\gamma_{a} \gamma_{e}}{\gamma_{a} \gamma_{c}-\gamma_{b}^{2}}
$$

$\Gamma_{23}^{G}=\frac{G_{31} \Gamma_{12}^{G}+G_{21} \Gamma_{31}^{G}+G_{21} \Gamma_{13}^{G}+G_{21} \sigma_{13}^{G}+G_{22} \sigma_{23}^{G}-G_{32}\left(\sigma_{22}^{G}-\sigma_{33}^{G}\right)-G_{31} \sigma_{12}^{G}-G_{33} \sigma_{32}^{G}}{G_{22}+G_{33}}$

$\gamma_{a}=\left(G_{11}+G_{22}\right)\left(G_{22}+G_{33}\right)-G_{31}^{2}$,

$$
\gamma_{b}=\left(G_{22}+G_{33}\right) G_{32}+G_{21} G_{31} \text {, }
$$




$$
\gamma_{c}=\left(G_{11}+G_{33}\right)\left(G_{22}+G_{33}\right)-G_{21}^{2}
$$

$$
\gamma_{d}=\left(G_{22}+G_{33}\right)\left[G_{11} \sigma_{12}^{G}-G_{21}\left(\sigma_{11}^{G}-\sigma_{22}^{G}\right)+G_{31} \sigma_{32}^{G}-G_{22} \sigma_{21}^{G}-G_{32} \sigma_{31}^{G}\right]+
$$$$
G_{31}\left[G_{21} \sigma_{13}^{G}+G_{22} \sigma_{23}^{G}-G_{32}\left(\sigma_{22}^{G}-\sigma_{33}^{G}\right)-G_{31} \sigma_{12}^{G}-G_{33} \sigma_{32}^{G}\right],
$$

$\gamma_{e}=\left(G_{22}+G_{33}\right)\left[G_{11} \sigma_{13}^{G}+G_{21} \sigma_{23}^{G}-G_{31}\left(\sigma_{11}^{G}-\sigma_{33}^{G}\right)-G_{32} \sigma_{21}^{G}-G_{33} \sigma_{31}^{G}\right]-$

$$
G_{21}\left[G_{21} \sigma_{13}^{G}+G_{22} \sigma_{23}^{G}-G_{32}\left(\sigma_{22}^{G}-\sigma_{33}^{G}\right)-G_{31} \sigma_{12}^{G}-G_{33} \sigma_{32}^{G}\right]
$$

$$
T_{\vec{p}}=\left(\begin{array}{ccc}
\cos \psi \cos \phi & \cos \psi \sin \phi & -\sin \psi \\
\sin \theta \sin \psi \cos \phi-\cos \theta \sin \phi & \sin \theta \sin \psi \sin \phi+\cos \theta \cos \phi & \cos \psi \sin \theta \\
\sin \theta \sin \psi \cos \phi+\sin \theta \sin \phi & \cos \theta \sin \psi \sin \phi-\sin \theta \cos \phi & \cos \psi \cos \theta
\end{array}\right)
$$

\section{References}

[1] Owen, J. M., Villumsen, J. V., Shapiro, P. R. and Martel, H., “Adaptive Smoothed Particle Hydrodynamics: Methodology. II.," The Astrophysical Journal Supplement Series, 116, 2, 1998, 155-209.

[2] Liu, M. B., Liu, G. R. and Lam, K. Y., "Adaptive Smoothed Particle Hydrodynamics for High Strain Hydrodynamics with Material Strength," Shock Waves, 15, 1, 2006, 21-29.

[3] Smeets, B., Odenthal, T., Vanmaercke, S. and Ramon, H., "PolygonBased Contact Description for Modeling Arbitrary Polyhedra in the Discrete Element Method," Computer Methods in Applied Mechanics and Engineering, 290, 2015, 277-289.

[4] Bai, L., Srikanth, N., Kang, G. and Zhou, K., "Influence of Third Particle on the Tribological Behaviors of Diamond-Like Carbon Films," Scientific Reports, 6, 2016, 38279-38290.

[5] Everaers, R. and Ejtehadi, M. R., "Interaction Potentials for Soft and Hard Ellipsoids," Physical Review E, 67, 4, 2003, 041710-041717.

[6] Babadi, M., Ejtehadi, M. R. and Everaers, R., "Analytical First Derivatives of the RE-Squared Interaction Potential," Journal of Computational Physics, 219, 2, 2006, 770-779.

[7] Volokitin, A. I., “Casimir Frictional Drag Force between a $\mathrm{SiO}_{2} \mathrm{Tip}$ and a Graphene-Covered $\mathrm{SiO}_{2}$ Substrate," Physical Review B, 94, 23, 2016, 235450-235457.

[8] Tullis, T. E., "Rock Friction and Its Implications for Earthquake Prediction Examined via Models of Parkfield Earthquakes," PNAS, 93, 9, 1996, 3803-3810.

[9] Toro, G. D., Goldsby, D. L. and Tullis, T. E., "Friction Falls Towards Zero in Quartz Rock as Slip Velocity Approaches Seismic Rates," Nature, 29, 6973, 2004, 436-439.

[10] Sang, L. V., Yano, A., Fujii, S., Sugimura, N. and Washizu, H., "Coarse-Grained Model for Spring Friction Study of Micron-Scale Iron by Smoothed Particle Hydrodynamics," EPL, 122, 2, 2018, 26004-26009.

[11] Maveyraud, C., Benz, W., Sornette, A. and Sornette, D., "Solid Friction at High Sliding Velocities: An Explicit Three-Dimensional Dynamical Smoothed Particle Hydrodynamics Approach," Journal of Geophysical Research, 104, B12, 1999, 28769-28788.

[12] Sang, L. V., Yano, A., Isohashi, A., Sugimura, N. and Washizu, H., "Smoothed Particle Hydrodynamics Study of Friction of the CoarseGrained $\alpha-\mathrm{Al}_{2} \mathrm{O}_{3} / \alpha-\mathrm{Al}_{2} \mathrm{O}_{3}$ and $\alpha-\mathrm{Fe}_{2} \mathrm{O}_{3} / \alpha-\mathrm{Fe}_{2} \mathrm{O}_{3}$ Contacts in Behavior of the Spring Interfacial Potential," Tribology International, 135, 2019, 296-304

[13] Sang, L. V., Yano, A., Isohashi, A., Sugimura, N. and Washizu, H., "Friction and Friction Heat of Micronscale Iron," Journal of Tribology, 142, 9, 2020, 091702- 091707.

[14] Heyliger, P., Ledbetter, H. and Kim, S., "Elastic Constants of Natural Quartz," Journal of the Acoustical Society of America, 114, 2, 2003, 644-650.

[15] Tang, Y., Fang, J., Yan, X. and Ji, H. F., "Fabrication and Characterization of $\mathrm{SiO}_{2}$ Microcantilever for Microsensor Application," Sensors and Actuators B: Chemical, 97, 1, 2004, 109113.

[16] Iwasawa, M., Tanikawa, A., Hosono, N., Nitadori, K., Muranushi, T. and Makino, J., "Implementation and Performance of FDPS: A Framework for Developing Parallel Particle Simulation Codes," Publications of the Astronomical Society of Japan, 68, 4, 2016, 54-73.

[17] Perfilyev, V., Moshkovich, A., Lapsker, I., Laikhtman, A. and Rapoport, L., "The Effect of Vanadium Content and Temperature on Stick-Slip Phenomena under Friction of $\mathrm{CrV}(\mathrm{x}) \mathrm{N}$ Coatings," Wear, 307, 1-2, 2013, 44-51.

[18] Maier, S., Sang, Y., Filleter, T., Grant, M., Bennewitz, R., Gnecco, E. and Meyer, E., "Fluctuations and Jump Dynamics in Atomic Friction Experiments," Physical Review B, 72, 24, 2005, 245418-245427.

[19] Horn, H. M. and Deere, D. U., "Frictional Characteristics of Minerals," Géotechnique, 12, 4 1962, 319-335.

[20] Deng, K. and Ko, W. H., "A Study of Static Friction between Silicon and Silicon Compounds," Journal of Micromechanics and Microengineering, 2, 1, 1992, 14-21.

[21] Kumar, A., Staedler, T. and Jiang, X., "Effect of Normal Load and Roughness on the Nanoscale Friction Coefficient in the Elastic and Plastic Contact Regime," Beilstein Journal of Nanotechnology, 4, 2013, 66-71.

[22] Deulin, E. A., Gatsenko, A. A. and Loginov, B. A., "Friction Force of Smooth Surfaces of $\mathrm{SiO}_{2}-\mathrm{SiO}_{2}$ as a Function of Residual Pressure," Surface Science, 433-435, 1999, 288-292.

[23] Nakatani, M. and Scholz, C. H., "Frictional Healing of Quartz Gouge under Hydrothermal Conditions: 1. Experimental Evidence for Solution Transfer Healing Mechanism," Journal of Geophysical Research, 109, B7, 2004, B07201-B07219.

[24] Chester, F. M., "Effects of Temperature on Friction: Constitutive Equations and Experiments with Quartz Gouge," Journal of Geophysical Research, 99, B4, 1994, 7247-7261.

[25] Logan, J. M., "The Progression from Damage to Localization of Displacement Observed in Laboratory Testing of Porous Rocks," The Geological Society of London, 289, 1, 2007, 75-87.

[26] Tsukizoe, T. and Sakamoto, T., "Friction between Hard Rough and Soft Smooth Surfaces," Bulletin of JSME, 19, 127, 1976, 54-60.

This paper is licensed under the Creative Commons Attribution-NonCommercial-NoDerivatives 4.0 International (CC BY-NC-ND 4.0) International License. This allows users to copy and distribute the paper, only upon conditions that (i) users do not copy or distribute such paper for commercial purposes, (ii) users do not change, modify or edit such paper in any way, (iii) users give appropriate credit (with a link to the formal publication through the relevant DOI (Digital Object Identifier)) and provide a link to this license, and (iv) users acknowledge and agree that users and their use of such paper are not connected with, or sponsored, endorsed, or granted official status by the Licensor (i.e. Japanese Society of Tribologists). To view this license, go to https://creativecommons.org/licenses/by-nc-nd/4.0/. Be noted that the third-party materials in this article are not included in the Creative Commons license, if indicated on the material's credit line. The users must obtain the permission of the copyright holder and use the third-party materials in accordance with the rule specified by the copyright holder. 